Pacific Journal of Mathematics

MEROMORPHIC FUNCTIONS WITH NEGATIVE ZEROS AND
POSITIVE POLES AND A THEOREM OF TEICHMULLER 


\title{
MEROMORPHIC FUNCTIONS WITH NEGATIVE ZEROS AND POSITIVE POLES AND A THEOREM OF TEICHMULLER
}

\author{
JACK WILLIAMSON
}

Let $\mathscr{M}_{\lambda}$ denote the class of meromorphic functions of finite order $\lambda$ whose zeros lie on the negative real axis and whose poles lie on the positive real axis. Let $\mathscr{T}_{\lambda}$ denote the class of functions belonging to $\mathscr{M}_{\lambda}$ whose zeros and poles are symmetrically located along the real axis.

In the study of certain aspects of the value distribution properties of meromorphic functions of order $\lambda<1$, the class $\mathscr{C}_{\lambda}, \lambda<1$, has recently been found to display certain striking and useful extremal properties, while earlier results on the subclass $\mathscr{T}_{\lambda}, \lambda<1$, have been important as a guide to the possible values of their Nevanlinna deficiencies. In this note the class $\mathscr{T}_{\lambda}, \lambda>1$, is studied and it is concluded that certain extremal properties displayed by $\mathscr{C}_{\lambda}$ for $\lambda<1$ do not extend to the case $\lambda>1$.

Introduction. This note is concerned with Nevanlinna's theory of meromorphic functions. We will assume familiarity with the standard notation and terminology of that theory. The order $\lambda$ and the lower order $\mu$ of a meromorphic function $f$ are defined by the familiar relations

$$
\lambda=\lambda(f)=\limsup _{r \rightarrow \infty} \frac{\log T(r, f)}{\log r} ; \mu=\mu(f)=\liminf _{r \rightarrow \infty} \frac{\log T(r, f)}{\log r} .
$$

In 1939, Teichmüller [12] proved

THEOREM A. Let $f \in \mathscr{I C}_{\lambda}$ for $0 \leqq \lambda<1$ and assume that the zeros $\left\{a_{n}\right\}$ and the poles $\left\{b_{n}\right\}$ of $f$ satisfy

$$
a_{n}=-b_{n} \quad(n=1,2, \cdots) .
$$

If

$$
u=1-\delta(0, f), v=1-\delta(\infty, f)
$$

then

$$
u=v \geqq \cos \left(\frac{\pi \lambda}{2}\right)
$$

Although the hypothesis (1) of Teichmüller's theorem is quite restrictive, the theorem is important as a guide to possible relations 
between pairs of deficiencies of meromorphic functions of order less than one. Indeed, A. A. Gol'dberg [7] later showed that (3) remains valid for any meromorphic function of order less than one whose zeros $\left\{a_{n}\right\}$ and poles $\left\{b_{n}\right\}$ satisfy

$$
\left|a_{n}\right|=\left|b_{n}\right| \quad(n=1,2, \cdots) .
$$

Then, in 1960, using an important lemma of Gol'dberg [7] on the extremal growth properties of the functions in the class $\mathscr{C}_{\lambda}, \lambda<1$, Edrei and Fuchs [4] characterized the possible values of the pair $(\delta(0, f), \delta(\infty, f))$ for meromorphic functions of order less than one. If $u$ and $v$ have the same meaning as in (2) then their result ${ }^{1}$ is

THEOREM B. Let $f$ be a meromorphic function of order $\lambda, 0<$ $\lambda<1$. Then, in addition to the trivial inequalities, $0 \leqq u \leqq 1$ and $0 \leqq v \leqq 1$, $u$ and $v$ satisfy

$$
u^{2}+v^{2}-2 u v \cos \pi \lambda \geqq \sin ^{2} \pi \lambda .
$$

If $u<\cos \pi \lambda$, then $v=1$; and if $v<\cos \pi \lambda$, then $u=1$. Further, all values $u$ and $v$ compatible with these restrictions are actually possible.

It is quite clear that Theorem B contains the results of Teichmüller-Gol'dberg as a special case.

Although the extension of Theorem B to functions of order greater than one having arbitrarily distributed zeros and poles seems an exceedingly difficult problem, its extension to the class $\mathscr{C}_{\lambda}, 1<\lambda<$ $\infty$, is suggested by recent results of Hellerstein and Shea [8] which characterize the possible values of the pair $(\Delta(0, f), \Delta(\infty, f))[\Delta(c, f)$ denotes the Valiron deficiency of the value $c$ for $f$ ] for functions $f$ in this class and by the "symmetry" of the results giving bounds for Valiron deficiencies and the results giving bounds for Nevanlinna deficiencies of functions in $\mathscr{C}_{\lambda}$ (compare e.g., Theorems 1 and 2 [10] and Theorems A and B [8]). In particular, Hellerstein and Shea [8] obtained the following complement of Theorem A.

THEOREM C. Let $f \in \mathscr{C l}_{\lambda}$ for some finite non-integral value of $\lambda$ and let $q$ be the integer determined by

$$
q<\lambda<q+1
$$

If

$$
X=1-\Delta(0, f), Y=1-\Delta(\infty, f)
$$

\footnotetext{
${ }^{1}$ Edrei [1] has sharpened Theorem B by replacing $\lambda$ with $\mu$, throughout.
} 
and (1) holds, then

$$
X=Y \leqq \begin{cases}\frac{\left|\cos \left(\frac{\pi \lambda}{2}\right)\right|}{q+1} & (q \text { even }) \\ \frac{\left|\cos \left(\frac{\pi \lambda}{2}\right)\right|}{q+2\left|\cos \left(\frac{\pi \lambda}{2}\right)\right|} & (q \text { odd }) .\end{cases}
$$

The main purpose of this note is to show that Theorem $\mathrm{C}$ does indeed suggest the correct extension of Theorem A.

For convenience denote by $\mathscr{T}_{\lambda}$ the class of functions belonging to $\mathscr{C}_{\lambda}$ whose zeros $\left\{a_{n}\right\}$ and poles $\left\{b_{n}\right\}$ satisfy (1). Moreover, in each of the following theorems we will assume that $\lambda$ satisfies (6) for some integer $q \geqq 1$ and that $k=2[(q+1) / 2]$. We then prove

Theorem 1. Let $f \in \mathscr{T}_{2}$ and assume that $u$ and $v$ are defined by (2). Then for any $\rho$ satisfying

$$
\mu \leqq \rho \leqq \lambda
$$

we have

$$
u=v \geqq \begin{cases}\frac{\left|\cos \left(\frac{\pi \rho}{2}\right)\right|}{2\left|\cos \left(\frac{\pi \rho}{2}\right)\right|+k-1} & (k-1 \leqq \rho \leqq k) \\ \frac{\left|\cos \left(\frac{\pi \rho}{2}\right)\right|}{k+1} & (k<\rho \leqq k+1) .\end{cases}
$$

THEOREM 2. Let $f \in \mathscr{T}_{2}$ and assume that $X$ and $Y$ are defined by (7). Then for any $\rho$ satisfying

$$
\mu \leqq \rho \leqq \lambda
$$

we have

$$
X=Y \leqq \begin{cases}\frac{\left|\cos \left(\frac{\pi \rho}{2}\right)\right|}{2\left|\cos \left(\frac{\pi \rho}{2}\right)\right|+k-1} & (k-1 \leqq \rho \leqq k) \\ \frac{\left|\cos \left(\frac{\pi \rho}{2}\right)\right|}{k+1} & (k<\rho \leqq k+1)\end{cases}
$$


THEOREM 3. Let $f \in \mathscr{T}_{\lambda}$ and let $N(r)$ denote the common value of $N(r, 0)$ and $N(r, \infty)$. Assume that

$$
\lim _{r \rightarrow \infty} \frac{N(r)}{T(r, f)}=L
$$

Then

$$
L= \begin{cases}\frac{\left|\cos \left(\frac{\pi \lambda}{2}\right)\right|}{2\left|\cos \left(\frac{\pi \lambda}{2}\right)\right|+k-1} & (k-1<\lambda<k) \\ \frac{\left|\cos \left(\frac{\pi \lambda}{2}\right)\right|}{k+1} & (k<\lambda<k+1)\end{cases}
$$$$
T(r, f)=(1+o(1)) r^{2} \psi(r) \quad(r \rightarrow \infty)
$$

and

$$
n(r, 0) \equiv n(r, \infty)=(\lambda L+o(1)) r^{2} \psi(r) \quad(r \rightarrow \infty)
$$

where $\psi(r)$ is a slowly varying function; i.e.,

$$
\lim _{r \rightarrow \infty} \frac{\psi(\sigma r)}{\psi(r)}=1 \text { for every } \sigma>1 \text {. }
$$

We remark that Theorem 2 sharpens Theorem $\mathrm{C}$ by taking into account the lower order of $f$ while Theorem 3 extends a tauberian result of Edrei and Fuchs [5, Theorem 1] to the functions $\mathscr{T}_{\lambda}, \lambda>$ 1. This latter observation together with Theorem 4 of [8] suggest that the tauberian result of Edrei and Fuchs may be valid for higher orders.

1. Basic lemmas. The proofs of Theorems $1-3$ depend on three lemmas which we now introduce. The first involves the notion of Pólya peaks.

Lemma 1. Let $G(t)$ be a real, continuous, non-decreasing, unbounded function defined for $t \geqq t_{0}>0$, of order $\lambda$ and finite lower order $\mu^{2}$. Then to each finite $\rho$ satisfying $\mu \leqq \rho \leqq \lambda$ corresponds an increasing, unbounded, positive sequence $\left\{r_{n}\right\}$ called a sequence of Pólya peaks of the first (second) kind, order, $\rho$ and a triple of positive sequences $\left\{\varepsilon_{n}\right\},\left\{r_{n}^{\prime}\right\}$, and $\left\{r_{n}^{\prime \prime}\right\}$ such that

$$
2 \lambda=\limsup _{t \rightarrow \infty} \frac{\log G(t)}{\log t}, \mu=\liminf _{t \rightarrow \infty} \frac{\log G(t)}{\log t}
$$




$$
\lim r_{n}^{\prime}=\lim \frac{r_{n}^{\prime \prime}}{r_{n}}=\infty ; \lim \varepsilon_{n}=\lim \frac{r_{n}^{\prime}}{r_{n}}=0
$$

and such that

$$
\begin{aligned}
& G(t) \leqq\left(1+\varepsilon_{n}\right)\left(\frac{t}{r_{n}}\right)^{\rho} G\left(r_{n}\right) \quad\left(r_{n}^{\prime} \leqq t \leqq r_{n}^{\prime \prime}\right) \\
& \left(G(t) \geqq\left(1-\varepsilon_{n}\right)\left(\frac{t}{r_{n}}\right)^{\rho} G\left(r_{n}\right) \quad\left(r_{n}^{\prime} \leqq t \leqq r_{n}^{\prime \prime}\right)\right)
\end{aligned}
$$

For a proof of this lemma the reader can consult [1], [2], [4], and [11].

The next lemma is due to Shea [11, Lemma 3].

LEMMa 2. Let $h(z)$ be an entire function having only negative zeros which is a finite or infinite product of primary factors of genus q. If

(1.3) $K_{q}(t, r, \beta)=\frac{(-1)^{q}}{\pi}\left(\frac{r}{t}\right)^{q+1} \frac{r \sin q \beta+t \sin (q+1) \beta}{t^{2}+2 t r \cos \beta+r^{2}}(q \geqq 0,|\beta|<\pi)$

then

$$
\frac{1}{\pi} \int_{0}^{\beta} \log \left|h\left(r e^{i \theta}\right)\right| d \theta=\int_{0}^{\infty} N(t, 0) K_{q}(t, r, \beta) d t
$$

and

$$
\lim _{\beta \rightarrow \pi^{-}} \int_{0}^{\infty} N(t, 0) K_{q}(t, r, \beta)=N(r, 0) .
$$

Moreover, if

$$
k=2\left[\frac{q+1}{2}\right]
$$

then

$$
\begin{gathered}
\int_{0}^{\infty} s^{\rho}\left\{K_{q}(s, 1, \beta)+K_{q}(s, 1, \pi-\beta)\right\} d s=\frac{\cos \left(\frac{\pi}{2}-\beta\right) \rho}{\cos \frac{\pi \rho}{2}} \\
(k-1<\rho<k+1, \quad 0<|\beta|<\pi) .
\end{gathered}
$$

We remark here that while (1.7) is not explicitly stated in [11], it is an easy consequence of an elementary contour integration and the fact that 


$$
\begin{aligned}
& K_{q}(s, 1, \beta)+K_{q}(s, 1, \pi-\beta)=\frac{2}{\pi} s^{-k} \frac{s^{2} \sin (k+1) \beta-\sin (k-1) \beta}{s^{4}-2 s^{2} \cos 2 \beta+1} \\
&(0<|\beta|<\pi) .
\end{aligned}
$$

The following is our main Lemma.

Lemma 3. Let

$$
h(z)=\prod_{n=1}^{\infty}\left(1-\frac{z}{a_{n}}\right) \exp \left[\sum_{\jmath=1}^{q} \frac{1}{j}\left(\frac{z}{a_{n}}\right)^{j}\right]
$$

denote a convergent Weierstrass product of genus $q \geqq 1$ having only negative zeros $a_{n}$, let

$$
g(z)=\frac{h(z)}{h(-z)}
$$

and let $k=2[(q+1) / 2]$. Then for each $r>0$ there exist functions $\alpha_{i}=\alpha_{i}(r) i=0, \cdots, k$ and $\chi=\chi(r)$ satisfying

$$
0 \leqq \alpha_{0} \leqq \frac{\pi}{2(k+1)}
$$

(ii) $\quad \frac{2 j-1}{2(k-1)} \pi \leqq \alpha_{j} \leqq \frac{2 j+1}{2(k+1)} \pi \quad j=1, \cdots, \frac{k}{2}$

(iii) $\alpha_{j}=\pi-\alpha_{k-j} \quad j=0, \cdots, k$

(iv) $\chi(r)=1$ if $\alpha_{0}=0$ and 0 otherwise

such that if $\alpha(r)=\left(\alpha_{0}, \cdots, \alpha_{k}\right)$, if

$$
H_{q}(s, \alpha(r))=\sum_{j=0}^{k}(-1)^{j}\left\{K_{q}\left(s, 1, \alpha_{j}\right)+K_{q}\left(s, 1, \pi-\alpha_{j}\right)\right\}
$$

and if

$$
N(t)=N\left(t, \frac{1}{g}\right)=N(t, g)
$$

then

$$
T(r, g)=\frac{1}{r} \int_{\tau}^{R} N(t) H_{q}\left(\frac{t}{r}, \alpha(r)\right) d t+2 \chi(r) N(r)+\eta(\tau, r, R)
$$

where

$$
0 \leqq \eta(\tau, r, R) \leqq\left\{\begin{array}{l}
B\left[\left(\frac{r}{\tau}\right)^{k-1} T(2 \tau, g)+\left(\frac{r}{R}\right)^{k} T(2 R, g)\right] \\
B\left[\left(\frac{r}{\tau}\right)^{k} T(2 \tau, g)+\left(\frac{r}{R}\right)^{k+1} T(2 R, g)\right]
\end{array}\right.
$$


holds whenever

$$
0<2 \tau \leqq r \leqq \frac{1}{2} R
$$

( $B>0$ is an absolute constant).

Further, if $\alpha_{0}^{*}, \cdots, \alpha_{k}^{*}$ denote any $k+1$ constants satisfying inequalities (1.10) (i)-(iii) then

$$
H_{q}\left(\mathrm{~s}, \alpha^{*}\right) \geqq \frac{k}{2 \pi} \frac{s^{4 k}}{1+s^{8 k}} \quad(s>0, s \neq 1)
$$

and

$$
T(r, g) \geqq \frac{1}{r} \int_{0}^{\infty} N(t) H_{q}\left(\frac{t}{r}, \alpha^{*}\right) d t+2 \chi^{*} N(r)
$$

where $\chi^{*}=1$ if $\alpha_{0}^{*}=0$ and 0 otherwise.

We omit the proof of this Lemma since it parallels so closely the proofs of Lemma 3.3 and Main Lemma of [10].

2. The growth of functions in the class $\mathscr{A}_{\lambda^{*}}$ In this section we make some observations concerning the relative growth of functions in the class $\mathscr{C l}_{2}$ which are necessary for the proofs of Theorems 1 and 2.

If $f \in \mathscr{M}_{\lambda}$ and $f(0)=1$, then, as is well known, we can write

$$
f(z)=e^{Q(z)} \frac{\Pi E\left(\frac{z}{a_{n}}, q\right)}{\Pi E\left(\frac{z}{b_{n}}, q\right)}=e^{Q(z)} g(z)
$$

where $E(u, q)$ is the Weierstrass primary factor of genus $q$, where $q$ is the smallest nonnegative integer for which

$$
\sum\left(\frac{1}{\left|a_{n}\right|^{q+1}}+\frac{1}{\left|b_{n}\right|^{q+1}}\right)<\infty
$$

and where $Q(z)$ is a polynomial of degree $d \leqq \lambda$. If $d>q, f$ is dominated by the exponential factor $e^{Q(z)}$ and it is easy to show that

$$
T(r, f) \sim T\left(r, e^{Q}\right) \sim \alpha r^{d} \quad(0<\alpha=\text { constant })
$$

and that

$$
\lim _{r \rightarrow \infty} \frac{N(r, 0)}{T(r, f)}=\lim _{r \rightarrow \infty} \frac{N(r, \infty)}{T(r, f)}=0 .
$$

Thus the asymptotic behavior of $T(r, f)$ and of the ratios $N(r, 0) / T(r, f), N(r, \infty) / T(r, f)$ is completely determined when $d>q$. Accordingly, there will be no loss of generality if, in the remainder of 
this section, we assume that $d \leqq q$. (Of course if, as in the hypotheses of Theorems $1-3$, we assume that $\lambda$ satisfies (6) then $d \leqq q$ is immediate.)

We will now obtain lower bounds on $T(r, f)$. To that end assume that $q \geqq 1$, set $k=2[(q+1) / 2], \Lambda(t)=N(t, 0)+N(t, \infty)$, and choose

$$
\mathscr{E}(r)= \begin{cases}{\left[-\frac{\pi}{2}, \frac{\pi}{2}\right]} & \text { if } k=4 \iota, \iota=1,2, \cdots \\ {\left[-\pi,-\frac{\pi}{2}\right] \cup\left[\frac{\pi}{2}, \pi\right]} & \text { if } k=4 \iota+2, \iota=0,1, \cdots\end{cases}
$$

Then

$$
\begin{aligned}
& {[T(r, f)=m(r, f)+N(r, \infty)} \\
\geqq & \left.\frac{1}{2 \pi} \int_{\mathscr{C}(r)} \log \left|f\left(r e^{i \theta}\right)\right| d \theta+N(r, \infty)\right] \\
& =\frac{1}{2 \pi} \int_{\check{c}(r)} \log \left|g\left(r e^{i \theta}\right)\right| d \theta+N(r, \infty)+\frac{1}{2 \pi} \int_{\mathscr{C}(r)} \operatorname{Re} Q\left(r e^{i \theta}\right) d \theta .
\end{aligned}
$$

To obtain a lower bound for the first integral on the right hand side of inequality (2.6), we observe that $f \in \mathscr{C}_{2}$ implies that $\left|g\left(r e^{i \theta}\right)\right|$ is an even function of $\theta$ and apply Lemma 2 to get

$$
\frac{1}{2 \pi} \int_{\mathscr{E}(r)} \log \left|g\left(r e^{i \theta}\right)\right| d \theta \geqq \frac{r^{k-1}}{2 \pi} \int_{0}^{r} \frac{\Lambda(t)}{t^{k}} d t-N(r, \infty)
$$

if $k=4 \iota, \iota=1,2, \cdots$, while

$$
\frac{1}{2 \pi} \int_{\mathscr{E}(r)} \log \left|g\left(r e^{i \theta}\right)\right| d \theta \geqq \frac{r^{k-1}}{2 \pi} \int_{0}^{r} \frac{\Lambda(t)}{t^{k}} d t+N(r, 0)
$$

if $k=4 \iota+2, \iota=0,1,2, \cdots$.

To estimate the second integral on the right hand side of inequality (2.6) write

$$
Q(z)=a z^{d}+\cdots \quad a \neq 0 .
$$

Thus,

$$
\operatorname{Re} Q\left(r e^{i \theta}\right)=|a| r^{d} \cos (\psi+d \theta)+o\left(r^{d}\right) \quad(r \rightarrow \infty)
$$

where $\psi=\arg a$. Since

$$
\frac{1}{2 \pi} \int_{\mathscr{\delta}(r)} \cos (\psi+2 j \theta) d \theta=0 \quad \text { for } j=1,2, \cdots
$$

and since $d \leqq q \leqq k$, it follows from (2.10) and (2.11) that

$$
\frac{1}{2 \pi} \int_{\mathscr{E}(r)} \operatorname{Re} Q\left(r e^{i \theta}\right) d \theta=0\left(r^{k-1}\right) \quad(r \rightarrow \infty) .
$$


Thus (2.6)-(2.8) and (2.12) imply that

$$
\frac{T(r, f)}{r^{k-1}} \geqq \frac{1}{2 \pi} \int_{0}^{r} \frac{\Lambda(t)}{t^{k}} d t+0(1) \quad(r \rightarrow \infty) .
$$

In view of the fact that

$$
\Sigma\left(\frac{1}{\left|a_{n}\right|^{k-1}}+\frac{1}{\left|b_{n}\right|^{k-1}}\right)=\infty .
$$

The integral in (2.13) tends to $\infty$ with $r$, so that

$$
\lim _{r \rightarrow \infty} \frac{T(r, f)}{r^{k-1}}=\infty \text { and } \mu(f) \geqq k-1 \text {. }
$$

Moreover, in view of the obvious inequalities

$$
T(r, g)=T\left(r, f e^{-Q}\right) \leqq T(r, f)+O\left(r^{d}\right) \leqq T(r, g)+O\left(r^{d}\right)
$$

(2.15) implies that

$$
T(r, f) \sim T(r, g) \text { if } d \leqq k-1 .
$$

Note that for $q$ odd, $k=q+1$, so that (2.17) is always true by virtue of our assumption on $d$.

We conclude this section with a final observation concerning functions in $\mathscr{T}_{\lambda}$. We will show that $f \in \mathscr{T}_{\lambda}, q(\geqq 2)$ even, and $Q(z)$ of degree $q$ imply that $\mu(f) \geqq q$.

To that end, we start from the well-known representation

$$
\log h(z)=(-1)^{q} \int_{0}^{\infty} n\left(t, \frac{1}{h}\right) \frac{z^{q+1}}{t^{q+1}(z+t)} d t \quad(|\arg z|<\pi)
$$

due to Valiron [13, p. 237], valid for any canonical product $h(z)$ of genus $q$, having only negative zeros. Thus, since $f \in \mathscr{T}_{\lambda}$ implies

$$
f(z)=e^{Q(z)} \frac{h(z)}{h(-z)}=e^{Q(z)} g(z)
$$

for some canonical product $h(z)$ of genus $q$, having only negative zeros, we have

$$
\log \left|f\left(r e^{i \theta}\right)\right|=\log \left|g\left(r e^{i \theta}\right)\right|+\operatorname{Re} Q\left(r e^{i \theta}\right)
$$

where, in view of (2.18)

(2.21) $\log \mid g\left(r e^{i \theta}\right) \|=2 \int_{0}^{\infty} \frac{n\left(s r, \frac{1}{h}\right)}{s^{q}} \frac{s^{2} \cos (q+1) \theta-\cos (q-1) \theta}{s^{4}-2 s^{2} \cos 2 \theta+1} d \theta$ $(0<|\theta|<\pi)$. 
Note that there exists $\varepsilon=\varepsilon(q)>0$, independent of $r$, such that

$$
\begin{gathered}
\log \left|g\left(r e^{i \theta}\right)\right|>0 \\
\text { for } \theta \in I_{q}=\left[-\frac{3 \pi}{2 q}-\varepsilon,-\frac{3 \pi}{2 q}+\varepsilon\right] \cup\left[\frac{3 \pi}{2 q}-\varepsilon, \frac{3 \pi}{2 q}+\varepsilon\right] .
\end{gathered}
$$

Since by assumption $Q(z)=a z^{q}+\cdots, a \neq 0, q(\geqq 2)$ even, we have

$$
\operatorname{Re} Q\left(r e^{i \theta}\right)=|a| r^{q} \cos (\psi+q \theta)+o\left(r^{q}\right) \quad(r \rightarrow \infty)
$$

where $\psi=\arg a$. Since $\cos (\psi+q \theta) \geqq 0$ for $\theta=-3 \pi / 2 q$ or for $\theta=$ $3 \pi / 2 q$, there exists a measurable subset $\mathscr{E}$ of $I_{q}$, independent of $r$, such that

$$
\cos (\psi+q \theta)>0 \text { for } \theta \in \mathscr{E} .
$$

It now follows from (2.21) - (2.24) that

$$
T(r, f) \geqq m(r, f) \geqq \int_{\mathscr{E}} \log \left|f\left(r e^{i \theta}\right)\right| d \theta \geqq K r^{q}(1+o(1)) \quad(r \rightarrow \infty)
$$

for some positive constant $K$, depending only on $q$. This clearly implies that $\mu(f) \geqq q$.

3. Proof of Theorems 1 and 2. Let $\rho$ be any number satisfying

$$
\mu \leqq \rho \leqq \lambda
$$

Since we are assuming $\lambda$ is non-integral, it is sufficient to prove (9) and (10) for $k-1<\rho<k+1, \rho \neq k$. The cases $\rho=k, k-1, k+1$ will then follow by continuity.

Proof of Theorem 1. Let $\sigma$ be any number satisfying

$$
\limsup _{r \rightarrow \infty} \frac{N(r)}{T(r, f)}<\sigma
$$

where $N(r)$ denotes the common value of $N(r, f)$ and $N(r, 1 / f)$. Then for $t_{0}\left(=t_{0}(\sigma)\right)$ sufficiently large we have

$$
N(r)<\sigma T(r, f) \quad r \geqq t_{0} .
$$

Denote by $\left\{r_{n}\right\}$ a sequence of Pólya peaks of the first kind, order $\rho$, for $T(r, f)$ and let $\left\{\varepsilon_{n}\right\},\left\{r_{n}^{\prime}\right\}$ and $\left\{r_{n}^{\prime \prime}\right\}$ be the associated sequences. Since

$$
\lim r_{n}^{\prime}=\lim \frac{r_{n}^{\prime \prime}}{r_{n}}=\lim \frac{r_{n}}{r_{n}^{\prime}}=\infty
$$


we can choose $n_{0}$ so large that for $n \geqq n_{0}$

$$
r_{n}^{\prime \prime} \geqq 4 r_{n} \text { and } \frac{1}{2} r_{n} \geqq r_{n}^{\prime} \geqq t_{0}
$$

Fix $n \geqq n_{0}$ and set

$$
\tau=\tau_{n}=r_{n}^{\prime}, \quad r=r_{n} \text { and } R=R_{n}=\frac{1}{2} r_{n}^{\prime \prime}
$$

With this choice of $\tau, r$ and $R, 2 \tau_{n} \leqq r_{n} \leqq(1 / 2) R_{n}$.

If $k-1<\rho<k$ and $q$ is odd, then $k-1=q$ and (2.17) implies that

$$
T(r, f) \sim T(r, g) \quad(r \rightarrow \infty) .
$$

If $k-1<\rho<k$ and $q$ is even, then (3.1) implies that $\mu(f)<k(=$ $q)$. Thus the concluding observation of $\S 2$ implies that $d g Q(z)=d \leqq$ $q-1=k-1$; hence, by (2.17), (3.6) is valid.

If, on the other hand, $k<\rho<k+1$ (i.e., $q$ even, $q=k$ ), then we can assume that

$$
r_{n}^{k}=o\left(T\left(r_{n}, f\right)\right)
$$

(cf. the proof of Lemma 1). Consequently, (2.16) implies that (3.6) is valid along the sequence $\left\{r_{n}\right\}$.

Thus, if $k-1<\rho<k+1, \rho \neq k$, the above remarks and Lemma 3 imply that

$$
\begin{aligned}
(1+o(1)) T\left(r_{n}, f\right)= & \frac{1}{r_{n}} \int_{\tau_{n}}^{R_{n}} N(t) H_{q}\left(\frac{t}{r_{n}}, \alpha\left(r_{n}\right)\right) d t+2 \chi\left(r_{n}\right) N\left(r_{n}\right) \\
& +\eta\left(\tau_{n}, r_{n}, R_{n}\right) \quad(n \rightarrow \infty) .
\end{aligned}
$$

Using (3.2), (1.16) and the nature of the sequence $\left\{r_{n}\right\}$ we find that

$$
\begin{aligned}
& \frac{1}{r_{n}} \int_{\tau_{n}}^{R_{n}} N(t) H_{q}\left(\frac{t}{r_{n}}, \alpha\left(r_{n}\right)\right) d t+2 \chi\left(r_{n}\right) N\left(r_{n}\right) \\
\leqq & \frac{\sigma}{r_{n}} \int_{r_{n}^{\prime}}^{r_{n}^{\prime \prime}} T(t, f) H_{q}\left(\frac{t}{r_{n}}, \alpha\left(r_{n}\right)\right) d t+2 \chi\left(r_{n}\right) \sigma T\left(r_{n}, f\right) \\
\leqq & \left(1+\varepsilon_{n}\right) \sigma T\left(r_{n}, f\right)\left\{\frac{1}{r_{n}} \int_{r_{n}^{\prime}}^{r_{n}^{\prime \prime}}\left(\frac{t}{r_{n}}\right)^{\rho} H_{q}\left(\frac{t}{r_{n}}, \alpha\left(r_{n}\right)\right) d t+2 \chi\left(r_{n}\right)\right\} \\
\leqq & \left(1+\varepsilon_{n}\right) \sigma T\left(r_{n}, f\right)\left\{\int_{0}^{\infty} s^{\rho} H_{q}\left(s, \alpha\left(r_{n}\right)\right) d s+2 \chi\left(r_{n}\right)\right\} .
\end{aligned}
$$

Using (1.7), (1.11) and (1.10) (iii), (iv) we find that 
(3.10)

$$
\begin{aligned}
& \int_{0}^{\infty} s^{\rho} H_{q}\left(s, \alpha\left(r_{n}\right)\right) d s+2 \chi\left(r_{n}\right)=\sum_{j=0}^{k}(-1)^{j} \frac{\cos \left(\frac{\pi}{2}-\alpha_{j}\right) \rho}{\cos \left(\frac{\pi \rho}{2}\right)} \\
\leqq & \frac{1}{\left|\cos \left(\frac{\pi \rho}{2}\right)\right|}\left\{2(-1)^{k / 2} \cos \left(\frac{\pi}{2}-\alpha_{0}\right) \rho+k-1\right\} .
\end{aligned}
$$

Thus from (3.8) - (3.10) we deduce that

$$
(1+o(1)) T\left(r_{n}, f\right)
$$

$$
\begin{array}{ll}
\leqq & \left(1+\varepsilon_{n}\right) \sigma T\left(r_{n}, f\right) \frac{1}{\left|\cos \left(\frac{\pi \rho}{2}\right)\right|}\left\{2(-1)^{k / 2} \cos \left(\frac{\pi}{2}-\alpha_{0}\right) \rho+k-1\right\} \\
& +\eta\left(\tau_{n}, r_{n}, R_{n}\right) \quad(n \rightarrow \infty) .
\end{array}
$$

If $k-1<\rho<k$, we use (1.14), (3.5), (3.6) and the nature of the sequence $\left\{r_{n}\right\}$ to get

$$
\begin{aligned}
0 \leqq \eta\left(\tau_{n}, r_{n}, R_{n}\right) & \leqq 2 B\left\{\left(\frac{r_{n}}{\tau_{n}}\right)^{k-1} T\left(2 \tau_{n}, f\right)+\left(\frac{r_{n}}{R_{n}}\right)^{k} T\left(2 R_{n}, f\right)\right\} \\
& \leqq 2^{k+2} B T\left(r_{n}, f\right)\left\{\left(\frac{r_{n}^{\prime}}{r_{n}}\right)^{\rho-k+1}+\left(\frac{r_{n}}{r_{n}^{\prime \prime}}\right)^{k-\rho}\right\} \\
& \leqq o\left(T\left(r_{n}, f\right)\right) \quad(n \rightarrow \infty) .
\end{aligned}
$$

If $k<\rho<k+1$, then we use (1.14), (3.7), and the obvious inequality

$$
T(r, g) \leqq T(r, f)+O\left(r^{k}\right)
$$

to get

$$
\begin{aligned}
0 \leqq & \eta\left(\tau_{n}, r_{n}, R_{n}\right) \leqq B\left\{\left(\frac{r_{n}}{\tau_{n}}\right)^{k} T\left(2 \tau_{n}, f\right)+\left(\frac{r_{n}}{R_{n}}\right)^{k+1} T\left(2 R_{n}, f\right)\right\} \\
& +O\left\{\left(\frac{r_{n}}{\tau_{n}}\right)^{k}\left(2 \tau_{n}\right)^{k}+\left(\frac{r_{n}}{R_{n}}\right)^{k+1}\left(2 R_{n}\right)^{k}\right\} \\
\leqq & 2^{k+2} B T\left(r_{n}, f\right)\left\{\left(\frac{r_{n}^{\prime}}{r_{n}}\right)^{\rho-k}+\left(\frac{r_{n}}{r_{n}^{\prime \prime}}\right)^{k+1-\rho}\right\}+o\left(T\left(r_{n}, f\right)\right. \\
& =o\left(T\left(r_{n}, f\right)\right) \quad(n \rightarrow \infty) .
\end{aligned}
$$

Combining (3.11) and (3.12), (3.13) we obtain

$$
\begin{aligned}
(1+o(1)) \leqq & \left(1+\varepsilon_{n}\right) \sigma \frac{1}{\left|\cos \left(\frac{\pi \rho}{2}\right)\right|}\left\{2(-1)^{k / 2} \cos \left(\frac{\pi}{2}-\alpha_{0}\right) \rho+\right. \\
& +k-1\}+o(1) \quad(n \rightarrow \infty) .
\end{aligned}
$$

For $k-1<\rho<k$, (1.10) (i) implies that 


$$
(-1)^{k / 2} \cos \left(\frac{\pi}{2}-\alpha_{0}\right) \rho \leqq(-1)^{k / 2} \cos \left(\frac{\pi \rho}{2}\right)=\left|\cos \left(\frac{\pi \rho}{2}\right)\right| .
$$

Thus for $k-1<\rho<k(3.14)$ and (3.15) imply that

$$
1 \leqq \sigma \frac{1}{\left|\cos \left(\frac{\pi \rho}{2}\right)\right|}\left\{2\left|\cos \left(\frac{\pi \rho}{2}\right)\right|+k-1\right\} \text {. }
$$

If $k<\rho<k+1$, it follows immediately from (3.14) that

$$
1 \leqq \sigma \frac{k+1}{\left|\cos \left(\frac{\pi \rho}{2}\right)\right|}
$$

The assertion from Theorem 1 now follows from (3.16) and (3.17) and the definition of $\sigma$.

Proof of Theorem 2. First we observe that if $k<\rho<k+1$; that is, if $q$ is even and $q<\rho<q+1$, then (10) is an immediate consequence of (8) and (3.1) since $|\cos (\pi \rho / 2)| /(k+1)$ is a decreasing function of $\rho$.

Thus the case $k-1<\rho<k$ remains. First we remark that if $\lambda_{1}$ and $\mu_{1}$ denote the order and lower order of $N(r)$ then, since $\lambda$ is non-integral, $\mu_{1} \leqq \mu \leqq \lambda=\lambda_{1}$.

Let $\left\{r_{n}\right\}$ be a sequence of Pólya peaks of the second kind order $\rho$ for $N(r)$ and let $\left\{\varepsilon_{n}\right\},\left\{r_{n}^{\prime}\right\}$ and $\left\{r_{n}^{\prime \prime}\right\}$ be the associated sequences. Set $\alpha_{0}^{*}=0, \alpha_{j}^{*}=(\rho-k+2 j) 2 \rho / 2 \pi j=1, \cdots, k / 2$ and use (1.16), (1.17) and (3.6) to obtain

$$
\begin{aligned}
& \left(1+o(1) T\left(r_{n}, f\right)=T\left(r_{n}, g\right)\right. \\
& \quad \geqq \frac{1}{r_{n}} \int_{r_{n}^{\prime}}^{r_{n}^{\prime \prime}} N(t) H_{q}\left(\frac{t}{r_{n}}, \alpha^{*}\right) d t+2 N\left(r_{n}\right) \quad(n \rightarrow \infty) .
\end{aligned}
$$

By the nature of the sequence $\left\{r_{n}\right\},(1.1)$ and the choice of $\alpha_{0}^{*}$, $\cdots, \alpha_{k}^{*}$

$$
\begin{aligned}
& \frac{1}{r_{n}} \int_{r_{n}^{\prime}}^{r_{n}^{\prime \prime}} N(t) H_{q}\left(\frac{t}{r_{n}}, \alpha^{*}\right) d t+2 N\left(r_{n}\right) \\
\geqq & \left(1-\varepsilon_{n}\right) N\left(r_{n}\right)\left\{\frac{1}{r_{n}} \int_{r_{n}^{\prime}}^{r_{n}^{\prime \prime}}\left(\frac{t}{r_{n}}\right)^{\rho} H_{q}\left(\frac{t}{r_{n}}, \alpha^{*}\right) d t+2\right\}
\end{aligned}
$$

$$
\begin{aligned}
& =\left(1-\varepsilon_{n}\right) N\left(r_{n}\right)\left\{\int_{r_{n}^{\prime} / r_{n}}^{\int_{n}^{\prime \prime} / r_{n}} s^{\rho} H_{q}\left(s, \alpha^{*}\right) d s+2\right\} \\
& =\left(1-\varepsilon_{n}\right) N\left(r_{n}\right)\left\{\int_{0}^{\infty} s^{\rho} H_{q}\left(s, \alpha^{*}\right) d s+2+o(1)\right\} \\
& =\left(1-\varepsilon_{n}\right) N\left(r_{n}\right)\left\{\frac{2\left|\cos \left(\frac{\pi \rho}{2}\right)\right|+k-1}{\left|\cos \left(\frac{\pi \rho}{2}\right)\right|}+o(1)\right\}
\end{aligned}
$$


Combining (3.18) and (3.19) we obtain

$$
\begin{aligned}
& (1+o(1)) T\left(r_{n}, f\right) \\
\geqq & \left(1-\varepsilon_{n}\right) N\left(r_{n}\right)\left\{\frac{2\left|\cos \left(\begin{array}{c}
\pi \rho \\
2
\end{array}\right)\right|+k-1}{\left|\cos \left(\frac{\pi \rho}{2}\right)\right|}+o(1)\right\} \quad(n \rightarrow \infty) .
\end{aligned}
$$

In view of (1.1), the case for $k-1<\rho<k$ now follows from (3.20) by dividing by $T\left(r_{n}, f\right)$ and letting $n \rightarrow \infty$.

4. Proof of Theorem 3. First, we observe that hypothesis (11) together with (9) and (10) implies that $\mu=\lambda$ and that $L$ is given by (12). The fact that $\mu=\lambda$ and that $\lambda$ satisfies (6) implies that

$$
r^{q}=o(T(r, g))
$$

and hence, in view of inequalities (2.16), that

$$
T(r, f) \sim T(r, g) .
$$

Conclusion (13) now follows from (4.1) and Lemma 3 in virtually the identical way in which the proof of Theorem 1 of [9] follows from Lemma 1 of [9] and (1.16) of [9]. Once (13) is established it follows easily from (11) that

$$
N(r, 0) \equiv N(r, \infty)=(L+o(1)) r^{2} \psi(r) \quad(r \rightarrow \infty),
$$

where $\psi(r)$ satisfies (15). A straightforward tauberian argument [see e.g. 5, §6] then yields (14).

\section{Concluding remarks.}

1. Let $q(\geqq 1)$ be a given positive integer, $k=2[(q+1) / 2], \mu$ and $\lambda$ any numbers satisfying

$$
k-1 \leqq \mu<\lambda \leqq q+1
$$

and

$$
\sigma(\rho)= \begin{cases}\frac{\left|\cos \left(\frac{\pi \rho}{2}\right)\right|}{2\left|\cos \left(\frac{\pi \rho}{2}\right)\right|+k-1} & (k-1 \leqq \rho \leqq k) \\ \frac{\left|\cos \left(\frac{\pi \rho}{2}\right)\right|}{k+1} & (k<\rho \leqq k+1) .\end{cases}
$$

Then, proceeding along the lines of $\S 6$ of [10], it is not difficult 
to construct functions $g(z)$ belonging to $\mathscr{T}_{\lambda}$, having lower order $\mu$, such that

$$
X=Y=\min _{\mu \leqq \rho \leqq \lambda} \sigma(\rho)<\max _{\mu \leqq \rho \leqq \lambda} \sigma(\rho)=u=v .
$$

The functions will be of the form

$$
g(z)=\frac{h(z)}{h(-z)}
$$

where $h(z)$ is a canonical product of genus $q$ having only negative zeros. In view of (6.2) such functions clearly show that Theorems 1 and 2 are best possible and also show that the bound on the lower order of functions in $\mathscr{M}_{\lambda}$ given by (2.15) is sharp.

2. In the introduction we alluded to the difficulty of extending Theorem B, the "ellipse" problem for meromorphic functions, to functions of order greater than one. While the functions in the class $\mathscr{A}_{\lambda}, \lambda<1$, are the "extremal" functions for the solution to the "ellipse" problem for meromorphic functions of order $\lambda<1$ (as indicated in the proof of Theorem $B$ and by the examples showing the best possible nature of inequality (5)), this does not appear to be the case if $\lambda>1$. Indeed, whatever the solution to the "ellipse" problem for functions of order $\lambda$, lower order $\mu \geqq 1$, estimates obtained by Edrei [1, Theorem 4a] (see also [3], Theorem 1) show that for such functions

$$
u+v \geqq \max _{\mu \leqq \rho \leqq \lambda} \frac{|\sin \pi \rho|}{A \rho+\frac{1}{2}|\sin \pi \rho|} \quad(0<A<12) ;
$$

hence, the "extremal" functions, of say regular growth (i.e., $\lambda=\mu$ ), would have to satisfy

$$
u+v \rightarrow 0 \text { as } \lambda \rightarrow q=\text { a positive integer . }
$$

However, as indicated by Theorem 1, the functions in $\mathscr{T}_{2}$ having regular growth satisfy

(5.6) $\lim \inf (u+v) \geqq 2 /(q+1)$ as $\lambda \rightarrow q=a$ positive, even integer .

Thus, the behavior of functions in $\mathscr{T}_{\lambda}$ for $\lambda$ near positive, even integers indicates that the class $\mathscr{C}_{2}$ is probably not the class of "extremal" functions for the solution to the "ellipse" problem for meromorphic functions of order $\lambda>1$.

\section{REFERENCES}

1. A. Edrei, The deficiencies of meromorphic functions of finite lower order, Duke Math. J., 31 (1964), 1-22.

2. - Sums of deficiencies of meromorphic functions, J. Analyse Math., 14 
(1965), 79-107.

3. A. Edrei and W. H. J. Fuchs, On the growth of meromorphic functions with several deficient values, Trans. Amer. Math. Soc., 93 (1959), 292-328.

4. - The deficiencies of meromorphic functions of order less than one, Duke Math. J., 27 (1960), 233-250.

5. - Tauberian theorems for a class of meromorphic functions with negative zeros and positive poles, Proc. Internat. Confer. in Function Theory, Erevan 1965, Nauka, Moscow, (1966), 339-358.

6. W. H. J. Fuchs, Théorie de l'approximation des fonctions d'une variable complexe, Montreal, 1968.

7. A. A. Gol'dberg, On deficiencies of meromorphic functions. Dokl. Akad. Nauk S.S.S.R.(N.S.), 98 (1954), 893-895.

8. S. Hellerstein and D. F. Shea, Bounds for the deficiencies of meromorphic functions of finite order, Proc. of Symposia in Pure Math., 11, Amer. Math. Soc., Providence, 1968.

9. S. Hellerstein, D. F. Shea and J. Williamson, A tauberian theorem characterizing the regularity of growth of a class of entire functions, Duke Math. J., 38 (1970), 489-499.

10. S. Hellerstein and J. Williamson, Entire functions with negative zeros and a problem of $R$. Nevanlinna, J. Analyse Math., 22 (1969), 233-267.

11. D. F. Shea, On the Valiron deficiencies of meromorphic functions of finite order, Trans. Amer. Math. Soc., 124 (1966), 201-207.

12. O. Teichmüller, Vermutugen und Satze uber die Werteilung gebrochener Funktionen endlicher Ordnung, Deutsche Math., 4 (1939), 163-190.

13. G. Valiron, Sur les fonctions entières d'ordre fini et d'ordre nul, et en particular les fonctions à correspondence régulière, Ann. Fac. Sci. Univ. Toulouse, (3) 5 (1913), 117-257.

Received September 4, 1970 and in revised form June 24, 1971.

UNIVERSITY OF HAWAII 


\section{PACIFIC JOURNAL OF MATHEMATICS}

\section{EDITORS}

\section{H. SAMELSON}

Stanford University

Stanford, California 94305

\section{R. HOBBY}

University of Washington

Seattle, Washington 98105

\section{J. DugundjI}

Department of Mathematics University of Southern California Los Angeles, California 90007

RICHARD ARENS

University of California

Los Angeles, California 90024

\section{ASSOCIATE EDITORS}
E. F. BECKENBACH
B. H. NeumanN
F. WOLF
K. YOSHIDA

\section{SUPPORTING INSTITUTIONS}

\author{
UNIVERSITY OF BRITISH COLUMBIA \\ CALIFORNIA INSTITUTE OF TECHNOLOGY \\ UNIVERSITY OF CALIFORNIA \\ MONTANA STATE UNIVERSITY \\ UNIVERSITY OF NEVADA \\ NEW MEXICO STATE UNIVERSITY \\ OREGON STATE UNIVERSITY \\ UNIVERSITY OF OREGON \\ OSAKA UNIVERSITY
}

\author{
UNIVERSITY OF SOUTHERN CALIFORNIA \\ STANFORD UNIVERSITY \\ UNIVERSITY OF TOKYO \\ UNIVERSITY OF UTAH \\ WASHINGTON STATE UNIVERSITY \\ UNIVERSITY OF WASHINGTON \\ AMERICAN MATHEMATICAL SOCIETY \\ NAVAL WEAPONS CENTER
}

The Supporting Institutions listed above contribute to the cost of publication of this Journal, but they are not owners or publishers and have no responsibility for its content or policies.

Mathematical papers intended for publication in the Pacific Journal of Mathematics should be in typed form or offset-reproduced, (not dittoed), double spaced with large margins. Underline Greek letters in red, German in green, and script in blue. The first paragraph or two must be capable of being used separately as a synopsis of the entire paper. The editorial "we" must not be used in the synopsis, and items of the bibliography should not be cited there unless absolutely necessary, in which case they must be identified by author and Journal, rather than by item number. Manuscripts, in duplicate if possible, may be sent to any one of the four editors. Please classify according to the scheme of Math. Rev. Index to Vol. 39. All other communications to the editors should be addressed to the managing editor, Richard Arens, University of California, Los Angeles, California, 90024.

50 reprints are provided free for each article; additional copies may be obtained at cost in multiples of 50 .

The Pacific Journal of Mathematics is published monthly. Effective with Volume 16 the price per volume (3 numbers) is $\$ 8.00$; single issues, $\$ 3.00$. Special price for current issues to individual faculty members of supporting institutions and to individual members of the American Mathematical Society: $\$ 4.00$ per volume; single issues $\$ 1.50$. Back numbers are available.

Subscriptions, orders for back numbers, and changes of address should be sent to Pacific Journal of Mathematics, 103 Highland Boulevard, Berkeley, California, 94708.

PUBLISHED BY PACIFIC JOURNAL OF MATHEMATICS, A NON-PROFIT CORPORATION

Printed at Kokusai Bunken Insatsusha (International Academic Printing Co., Ltd.), 270, 3-chome Totsuka-cho, Shinjuku-ku, Tokyo 160, Japan. 


\section{Pacific Journal of Mathematics}

\section{Vol. 42, No. $3 \quad$ March, 1972}

Catherine Bandle, Extensions of an inequality by Pólya and Schiffer for vibrating membranes ................................ 543

S. J. Bernau, Topologies on structure spaces of lattice groups.......... 557

Woodrow Wilson Bledsoe and Charles Edward Wilks, On Borel product measures .......................................

Eggert Briem and Murali Rao, Normpreserving extensions in subspaces of

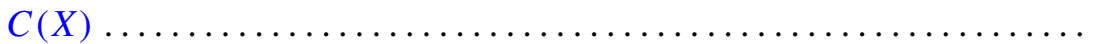

Alan Seymour Cover, Generalized continuation.................. 589

Larry Jean Cummings, Transformations of symmetric tensors .......... 603

Peter Michael Curran, Cohomology of finitely presented groups .......... 615

James B. Derr and N. P. Mukherjee, Generalized quasicenter and

hyperquasicenter of a finite group ...................... 621

Erik Maurice Ellentuck, Universal cosimple isols .................. 629

Benny Dan Evans, Boundary respecting maps of 3-mainfolds .......... 639

David F. Fraser, A probabilistic method for the rate of convergence to the

Dirichlet problem .................................. 657

Raymond Taylor Hoobler, Cohomology in the finite topology and Brauer

groups ..................................... 667

Louis Roberts Hunt, Locally holomorphic sets and the Levi form ........ 681

B. T. Y. Kwee, On absolute de la Vallée Poussin summability............ 689

Gérard Lallement, On nilpotency and residual finiteness in semigroups .... 693

George Edward Lang, Evaluation subgroups of factor spaces........... 701

Andy R. Magid, A separably closed ring with nonzero torsion pic ....... 711

Billy E. Rhoades, Commutants of some Hausdorff matrices ............. 715

Maxwell Alexander Rosenlicht, Canonical forms for local derivations . . . . 721

Cedric Felix Schubert, On a conjecture of L. B. Page ................ 733

Reinhard Schultz, Composition constructions on diffeomorphisms of $S^{p} \times S^{q}$

J. P. Singhal and H. M. (Hari Mohan) Srivastava, A class of bilateral generating functions for certain classical polynomials ....

Richard Alan Slocum, Using brick partitionings to establish conditions which insure that a Peano continuum is a 2-cell, a 2-sphere or an annulus...

James F. Smith, The p-classes of an $H^{*}$-algebra ...

Jack Williamson, Meromorphic functions with negative zeros and positive

poles and a theorem of Teichmuller ................. 